\title{
A Simulation-Based Decision Support Method for Modular Product Architecture Alternatives
}

\author{
F. M. Seiler ${ }^{1}$, J. Kuhl ${ }^{1}$, D. Krause ${ }^{1}$ \\ ${ }^{1}$ Institute for Product Development and Mechanical Engineering Design \\ Hamburg University of Technology (TUHH)
}

\begin{abstract}
With a constantly increasing market competition leading to high degrees of product individualization and customization, developing product architectures, which still offer competitive advantages is crucial to success. For the concept of modularization supplying one solution to this issue, there are many modularization approaches available. As these all lead to different modular product architecture alternatives when being applied, the decision of which alternative to finally implement becomes increasingly difficult with more and more complex product architectures. With this contribution, we propose a simulation-based approach using model-based systems engineering as a consistent root data system for product configuration systems in order to address both customer- and company perspectives for analysing the architecture alternatives' performances. Considering the multidimensional environment, a hyperspace algorithm for expressing individual architectures as geometric representations is used. Applying the simulation method to a medical stent as exemplary product, the implementation, results and capabilities of such a simulation is displayed.
\end{abstract}

Keywords: product architecture, dependencies, simulation, performance analysis

\section{Introduction}

With globalization representing one of today's major trends, global markets face a continuously increasing competition within the field of production companies, resulting into an ever-increasing race concerning production cycles, cost and material effort. In order to cope with this situation, suppliers and end-product manufacturers rely increasingly on product specialisation to meet the demand for customised machine or product systems. Unfortunately, product customisation inhibits many internal standardisation and optimisation possibilities. In most cases, a growing external variety, meaning the variety of offered product variants, possess a large degree of component as well as process diversity and structural complexity, which can be assumed as internal variety [Kra18], since product variants are manufactured in very small batch sizes, sometimes even as single-unit products. This combination creates a large diversity within business processes, resulting in an increase of costs in all product life phases. In order to ensure the individual company's competitiveness, managing these variants with their product- and process structure is one of the key tasks to challenge [Kra18]. Within the concept of modular product architectures (MPA) supplying one possible solution to reduce the internal variety and thus costs while still keeping the external variety towards the customer constant, researchers have provided different approaches in order to meet these opposing demands. Due to the large amount of individual methods, many different MPA alternatives can be 
methodically derived for a company's current situation. All MPA consist of a large number of modules, components, constraints, linkages and sets of parameters which are individually influenced by internal and external factors from the company's and customer's business environment. Therefore, a large amount of data needs to be processed, stored and analysed when trying to differentiate the individual architectures' effects and performance with regard to the underlying circumstances. This can hardly be done objectively by manual means. Hence, a proper modular architecture data management as a basis for the performance analysis of different architecture alternatives with respect to individual customer and company requirements is needed, both for research and practical application.

This contribution proposes a methodical approach for a detailed, simulation-based MPA alternative analysis, Thus, the decision process about which MPA alternative should finally be implemented as the result of an ongoing modularization process is supported. In order to come up with a properly resilient solution, a close consideration towards not only the product perspective but also the customer and company perspective is taken. The proposed method is described using the product example of medical stents with its underlying product architecture and corresponding set of dependencies and constraints.

\section{State of the Art}

When it comes to the methodical evaluation of modular product architectures (MPA), the pertinent literature distinguishes between four different categories of methods [Win19]. First of all, there are methods strictly focusing on structural indices such as cluster indicators, or the degree of modularity, etc. [Salv07]. The second category focuses on the economic success of product structures, evaluating lead times, revenue or the time-tomarket index. Combining both approaches, universal methods combine technical and product-strategic dimensions [Höl06], but still do not consider specific product processes. Taking this approach class one step further [Kvi10], the fourth category consists of comprehensive methods, including specific methods for the planning, development and assessment for product families in general, taking as well product-strategic and functional indications into account. Simpson et al. [Sim12] provide one approach within this category with the Integrated Approach to Product Family Design, as well as Krause et al. [Kra18] with the Integrated PKT-approach for the development of modular product families. Considering these different approaches for MPA development and evaluating their applicability for analysing of the developed modular product architectures' performance, several benefits and deficiencies to the currently available methods for the evaluation of MPA become apparent. As assessing modular product architectures' performances comes down to a compared analysis of both, the customer and the company perspective, tradeoffs between those are inevitable. This is further emphasized when taking the complete set of customer-relevant properties to be fulfilled by a product variant into account [Win19]. With these both perspectives in mind, Harlou et al. [Har06] provide an approach to conjointly analyse the intercorrelation between structural requirements and customer perspectives, but keep this approach at a general level with a lacking degree of detailing. Kvist tackles this issue with integrating further detail to their method, but only focuses on supply chain integration [Kvi10]. This still results in a missing consideration of all other life phases, such as R\&D or sales. A different approach taking all life phases into account is provided by Ericcson and Erixon [Eri99], including performance metrics based upon 
modularization drivers from the individual life phases with a strong focus on the product strategic view. Nevertheless, when it comes to compare different MPA alternatives, this approach does not provide a suitable solution. In order to provide an adequate analysis result, a set of multiple influencing factors which discretely describe the underlying company- and customer situation needs to be included. There are several different methods performing a multi-dimensional analysis like e.g. binary comparison or cost-benefit analysis etc. A close comparison and description of these methods is done by Wartzack et al. [War13]. One major deficiency all these different approaches have in common is that they are only regarding either the weighting of multiple factors or the comparison of two individual factors, but not both. At this specific point, the research of Windheim [Win19] is based, offering an approach for cooperative decision making in modular product family design. This method supplies an approach for the differentiation between MPA alternatives, taking both the customer as well as the company perspectives into account whilst including multiple factors like e.g. the by Ripperda et al. [Rip19] proposed variety induced complexity costs and further cost effects linked to the activity-based costing method simultaneously. Furthermore, the company's organizational structure can be integrated. Nevertheless, as Windheim's approach as well as the tools provided are strongly linked to the decision-making process itself, multiple criteria are considered, but still with using a single-layer correlation approach. Additionally, Yu et al. [Yu 07] provide one of the earliest automated algorithms to develop modular architectures using DSMs based on the minimum description length principle in information theory. Nevertheless, multi-layer dependencies between criteria, with one effect impacting several others and thereby triggering further changes are not included. Furthermore, this approach aims minimizing the above-mentioned trade-offs without considering the potential effects, benefits or disadvantages linked to alternative trade-off degrees. This leads potentially not to the most performant MPA with respect to the company's benefits, as sometimes even slightly increased or decreased trade-offs can result in a major impact to the MPA alternative's overall performance.

Therefore, this contribution tries to fill this gap by presenting a methodical, simulationbased approach, tackling the situation by varying alternative MPA. As this comes with the need of analysing large amounts of data, especially with different data types, structures, logical ontologies and corresponding functions and dependencies, reducing the potential for inconsistencies [Han18] is key for providing a reliable simulation output. The approach of model-based systems engineering (MBSE) provides one suitable solution to this issue, as its meta-model structure allows for combining geometric and structural information with logical and functional background in a central root data core, consistently deriving information from this central core only when needed [Sei19].

\section{Introduction to the used Product Example: Flow Diverter}

In order to explain the proposed method, the applied medical product example is of reduced complexity in order to focus on the methodical approach's functionality. In this section, the used product example and the necessary background information is described.

An aneurysm is a pathological dilatation of a blood vessel, which preferentially occurs at the sites of division, the bifurcations, of the blood vessel tree [Gas05]. Various sections of 
the brain vessels are affected with varying degrees of frequency (see Figure 1) [Gas05], [Hac10]. To prevent rupture of an aneurysm, which can lead to life-threatening bleeding, an aneurysm is treated with a flow diverter (FD) under certain conditions [Gas05]. An FD is a braided stent that is implanted into the blood vessel in front of the aneurysm, reconstructing the vessel and altering the blood flow into the aneurysm so that it can regress to the aneurysm [Dur11]. A prerequisite for this is that the FD must adapt as completely as possible to the wall of the blood vessel, which is why the diameter of the FD should be at least as large as the diameter of the vessel. In addition, the FD must be as short as possible, but must cover the aneurysm sufficiently (at least $5 \mathrm{~mm}$ before and after the aneurysm) and, if possible, not begin or end in a vessel curve [Dur11]. To meet these requirements, various manufacturers offer Flow Diverter in different length and diameter gradations, from which the treating physician can select a suitable size (Figure1)

(a)

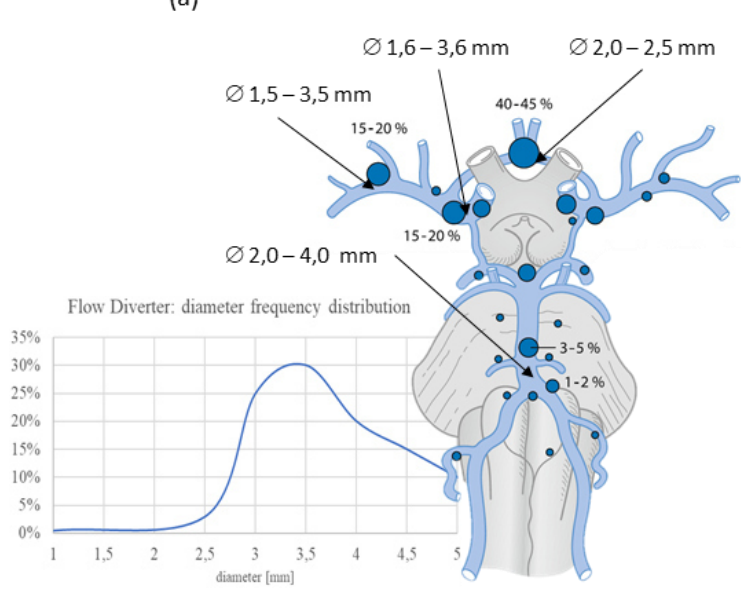

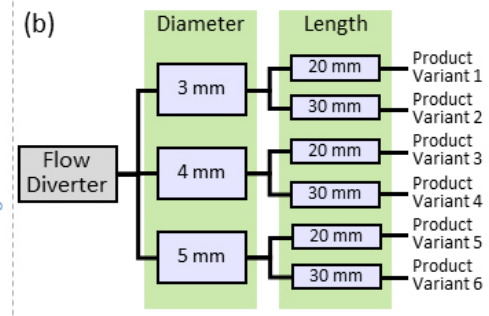

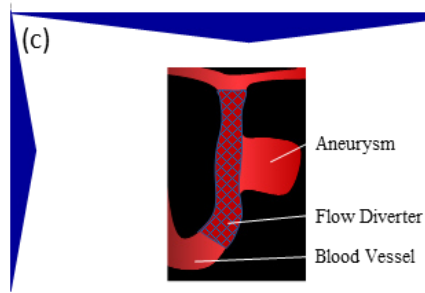

Figure 9: Frequency of saccular aneurysms on arteries with different diameters (according to [Hac10]) (a) as well as fictitious product family of a flow diverter (b) for the treatment of an aneurysm (according to [Kuh20]), (c)

When determining the external variety, a company is faced with the question of the degree of differentiation with which the FD should be offered in order to be able to provide the best possible care for the patient anatomy while at the same time being able to handle variant-induced complexity. The material and manufacturing costs hardly differ from variant to variant, but an expensive tool must be purchased for each diameter gradation. The device length is mainly controlled by parameters of the machine program. Based on the vessel diameters and occurrence probabilities listed in the literature, a multidimensional simulation method based upon a product configuration system can be applied in order to investigate and evaluate different MPA alternatives with varying aspects, such as for example offering the entire range of possible vessel diameters or concentrate only on the frequently occurring diameters with varying length expressions. 


\section{Multi-Dimensional Simulation: Data modelling and processing}

Based on the Flow Diverter example, the proposed MPA alternative decision method is described as follows. Within the process of modularization, different MPA alternatives can be developed and a decision of the most suitable alternative for the customer as well as for the company needs to be made. The presented approach aims to provide a repeatable, quantitative and objective decision support by taking multiple interconnected analysis dimensions into account. Figure 2 gives a schematic overview about the general methodical set-up.

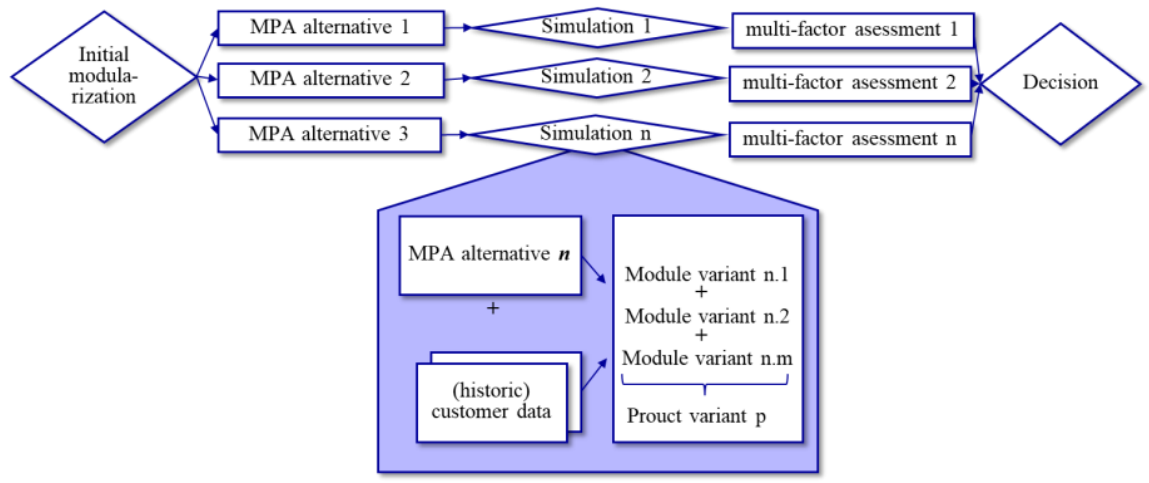

Figure 2: Schematic overview of decision support simulation method

The first step is to transform an unstructured product architecture into a modular product architecture, using modularization methods during the initial modularization phase. As already described, various methods lead to differing MPA alternatives. On this general level, it is not crucial to define the individual modularization method. The resulting MPAs $1 . . . \mathrm{n}$ of the initial modularization phase define the total sum of alternatives, amongst which the most performant is to be determined. The present processes come down to experts using implicit knowledge and a limited number of analysing factors, like e.g. the number of standard components within the individual alternative, in order to conclude a qualitative result for the most performant alternative. As those MPA alternatives are composed of a vast amount of data with - depending on the product's complexity - a large amount of dependencies and constraints as well as the company's business and customer environment being described by multiple different variables needs to be considered, a computer-based simulation seems to be the only reasonable way to process these large data volumes.

Having determined all different alternatives, simulating these alternatives while considering both, customer and business environmental factors, is the next step to create a basis for the subsequent multi-factor assessment phase. In order to analyse an individual MPA alternative's, using a product configuration system as the key simulation component provides one possible solution to intuitively but consistently integrating both perspectives into the analysis process. This product configuration system is used to determine the most appropriate product variant within the individual product architecture alternative for individual sets of customer requirements. By using multiple sets of customer requirements, which can e.g. be generated by deriving them from historic customer specification books 
this configuration process is performed multiple times, leading to an increasing dataset containing matching pairs of corresponding customer requirements and product variants for one individual modular product architecture alternative. These multiple configuration processes are then repeated, each time with a different architecture alternative as a data basis for the product configuration system, generating a different data set of corresponding customer requirements to product variants for each architecture alternative. Having generated these data sets for every individual MPA, they can be analysed during the next methodical step using a multi-factor analysis. In general, every company and every MPA need to be analysed considering different aspects. These individual aspects can then be combined using a multi-dimensional Hyperspace algorithm, which basically expresses MPA alternatives as geometric areas in a multi-dimensional spatial area [Sei20]. By integrating and minimizing these spatial areas, the most performant MPA alternative can be identified [Sei20]. This leads to the final step, providing an objective performance index for the modularization team when deciding about which MPA alternative to implement.

In order to exemplarily show the application of the described method, the Flow Diverter product example is used, where the FD is treated as one module of an overall treatment system. According to the literature analysis (compare section 3), the underlying product family corresponds to two customer-relevant properties, first to match the vessel diameter and secondly, to patch the blood vessels defect in length. As the overall variance of the diameter and length expressions for the aneurysms reach approximately from $2.0 \mathrm{~mm}$ to $4.0 \mathrm{~mm}$ in diameter and from $10 \mathrm{~mm}$ to $25 \mathrm{~mm}$ in length (see figure 1), an implemented product family needs to be assessed about the degree of its compliance with this anatomic situation. As described above, the diameter for the matching Flow Diverter needs to either equal the vessel's diameter or be slightly larger, while its length needs to correspond to the vessel length along the vessel plus approximately $10 \mathrm{~mm}$ for properly patching the defect and being reliably anchored. The data source for the simulation and therefore the configuration system is provided by a MBSE root data core, providing a suitable consistency for the methodical process. Therefore, the two described constraints for identifying the most adequate product variant for the underlying anatomic defect are modelled as a parametric diagram in the MBSE environment as shown in figure 3. In the presented case study, we use Cameo Systems Modeler (CSM) by NoMagic, Inc.

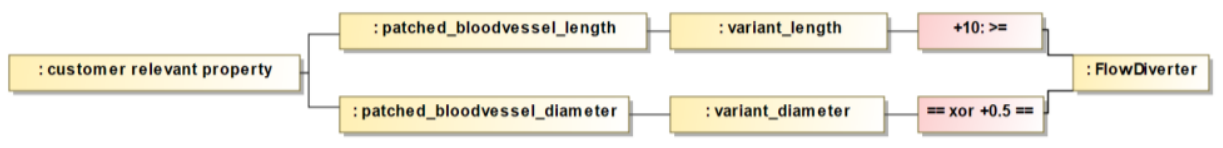

Figure 3: MBSE constraint parameter diagram for product configuration system

These constraints individually address their corresponding customer-relevant properties and can be exported from CSM in order to be dynamically implemented into the configuration system's product variant determination algorithm, enabling a fast and consistent possibility of implementing the product variant determination semantics. As for the MPA alternatives' implementation themselves, a data structure expressing both, customer-relevant properties as well as the product variants' modules with their internal variety, is needed. One possible solution is offered by an adapted network diagram from the sales life phase, connecting customer-relevant properties to individual components and 
clustering them to individual modules [Kra18]. Including this structure in CSM, BlockDefinition-Diagrams (BDDs) are used in order to model and display the MPA alternatives. The following figure 4 displays the network diagram for the used product example.

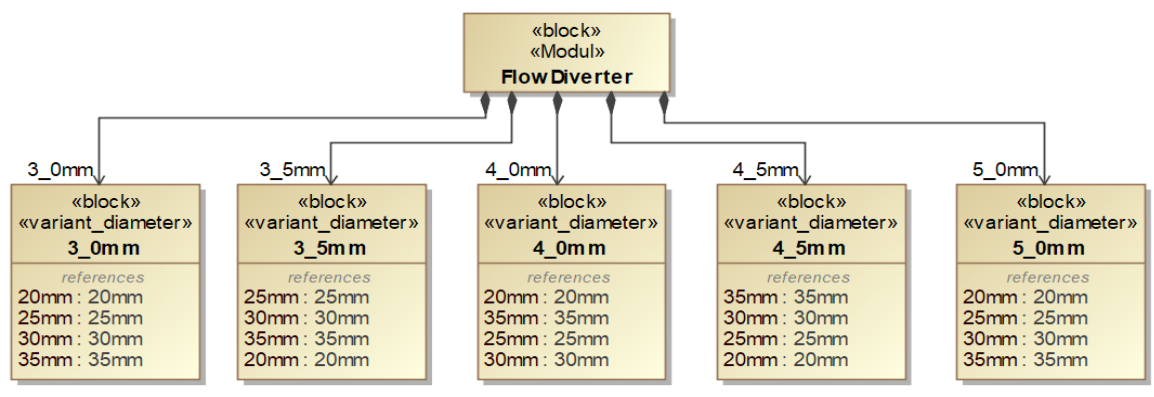

Figure 4: MBSE network diagram with module variants

The customer-relevant properties are connected in a separate, but centrally linked BDD to their corresponding module. In order to integrate this MPA into the configuration system as the underlying database, we use a multiple set of dependency matrices for exporting the connections of customer-relevant properties to modules, the connections of modules to module variants as well as their internally variant expressions. The following figure 5 displays three dependency matrices, each of them representing an individual MPA alternative, which have been developed methodically during the initial modularization phase. Each MPA alternative differs in the extent of offered Flow Diverter diameter and length expressions, being marked by the "arrow"-symbol, as a predefined dependency.

\section{MPA alternative 1}

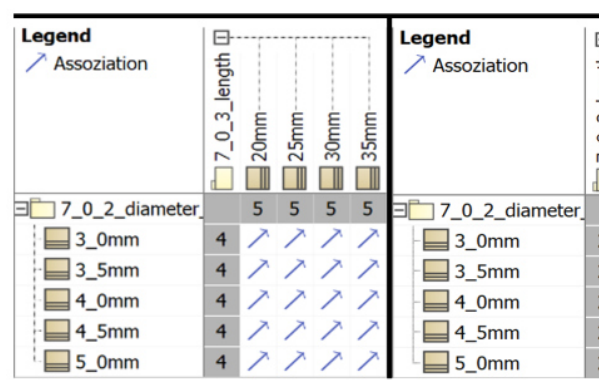

MPA alternative 3

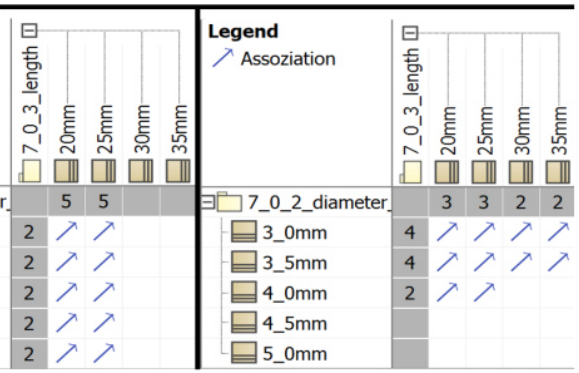

Figure 5: MPA alternatives; Dependency matrices with varying length and diameter expressions

MPA alternative 1 represents the maximum range of offered diameter and length expressions with a total amount of 20 product variants, whereas alternatives two and three offer only ten product variants. The second MPA offers the complete diameter range with two length expressions each, whereas MPA alternative three offers only three diameter ranges with different lengths. When analysing these alternatives, the performance indication dimensions need to be defined. One important factor called configuration depth [Sei19] describes how many specific customer requests can be met by the individual MPA 
alternative. Furthermore, the order-lead time for the Flow Diverter batch is considered as a second dimension. The calculation of the overall order-lead time is based upon diameter changes or length variations, where diameter variants impact five times stronger than length variations due to an increased effort in tool production and changing and re-setting of the Flow Diverter production system. Therefore, an MPA with more diameter variations than length variation should return a longer order-lead time than an alternative with only variations in lengths. The introduced three different MPA alternatives have then been simulated using the proposed method. The results, mapped as 3D-spatial areas as shown in figure 5, display the graphical representation of the hyperspace algorithm.

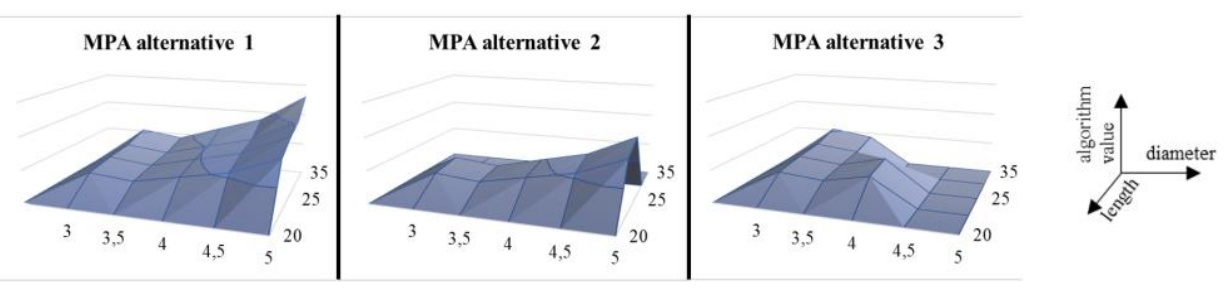

Figure 5: Graphical representation of simulation results for three MPA alternatives

With the $\mathrm{X}$-Axis referring to matched diameter ranges and the Y-Axis mapping the corresponding length variations, the overall internal product architecture variety is displayed. The Z-Axis describes a combined, dimension-less value of configuration depth and normalized order-lead time, using Euclidian multi-dimensional scaling for being able to display the simulation result in three dimensions [Sei20]. When using the hyperspace algorithm, the individual spatial areas get interpolated in order to return a mathematical function, which is then integrated over the interval reaching from zero to the maximum expression amongst all alternatives. The resulting minimal spatial area value indicates the most performant MPA alternative [Sei20]. In this case, when closely regarding the three displayed graphs, it becomes obvious that alternative 1 refers to the largest spatial area. As this alternative expresses all diameter and length variations, its configuration depth reaches $100 \%$, but also comes with the largest overall order lead time. This order lead time is only marginally reduced within alternative 2 , as there still are all five diameter variants considered, but with only two different length variants. The more crucial aspect within alternative 2 is the reduced configuration depth, which just exceeds $70 \%$, a value given by the configuration system used for the calculation of the mapped algorithm value. Within alternative 3, only three diameters are expressed. Although expressing the same amount of product variants as alternative 2, the configuration depth of alternative 3 is significantly higher. This is due to the fact, that most of the customer-requests are set within the lower diameter range (compare figure 1). This leads to the conclusion, that the information about fewer diameter variations and more length variations supplies sufficient information for identifying the most performant MPA alternative. Nevertheless, the individual arrangement of which diameter and length variation to offer is still crucial. In order to display the potential of the simulation for not only identifying such tendencies but also to derive the high-impact product variants, the following figure 6 displays a fourth MPA alternative and the corresponding simulation result. 


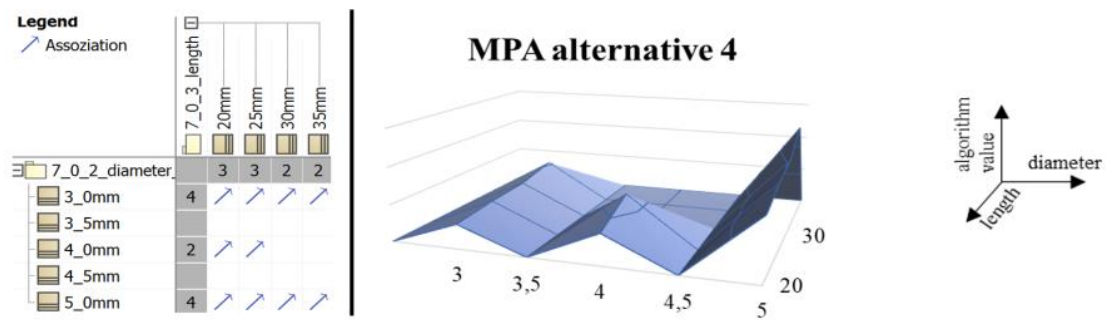

Figure 6: Database and simulation result of MPA alternative 4, displaying impact variants

MPA 4 is closely linked to MPA 3, but includes other variants of diameter arranged at the margins of the range $(5.0 \mathrm{~mm}$ diameter instead of $3.5 \mathrm{~mm})$. The resulting, simulated spatial area is significantly larger than with the above described MPA alternative 3. Furthermore, the product variant with a diameter of $5.0 \mathrm{~mm}$ and a length of $35 \mathrm{~mm}$, representing the largest diameter with the greatest length variation, is connected to a strongly impacting performance value, representing a large negative impact to the MPA alternative's performance in comparison to the added value. This results from the amount of customer requests for this product variant, as only $10 \%$ need this diameter and amongst them 5\% require the corresponding length expression. Opposed to that, the length range linked to a diameter of $3.0 \mathrm{~mm}$ apparently has a significantly positive impact on the MPA alternative's performance. All in all, MPA alternative 3 seems to represent the most performant one. This mathematically derived performance index can then be used by the modularization team to finally decide about which MPA alternative to implement.

\section{Discussion and Outlook}

With its capability of representing both, customer and company perspectives, and being able to efficiently and repetitively process large data amounts, the use of a configuration system as core simulation element seems to be a reasonable choice. Considering the data collection for the root data core, a high practicability is given due to the visual support supplied by e.g. the dependency matrices. Nevertheless, the analysis dimension definition needs to be adapted for other product and customer environments. In order to keep this particular step methodically accurate, further research needs to be done. One possible solution can be provided by deriving the analysis dimensions from an Impact Model, representing a methodical approach of analysing and modelling the impacting factors to the product-; company-; and customer environment [Hac20], [Sch20]. Furthermore, a simulation like we proposed can just offer an objective decision support, which still needs to be revised by the expert modularization team. Furthermore, the MBSE-based configuration systems can be used to support the analysis and decision-making process in product individualization. MPA alternatives, which provide defined customer individually adaptable modules, can be investigated. With the help of the configuration system, it can be examined how the configuration depth is thereby influenced and whether there is sufficient added value for the customer to accept the increased internal variety that can be managed through flexible product and process structures. As the simulation generically addresses the data structure, the proposed process is completely automated apart from the MPA alternative generation. Using automated methods such as provided by $\mathrm{Yu}$ et al. 
[Yu07], the automation degree can be increased. All in all, this contribution shows the requirements, implementation and results of this simulation-based method, providing a multi-dimensional, interconnected decision support for MPA alternative differentiation.

\section{References}

[Eri99] Ericsson, A.; Erixon, G.: "Controlling design variants: Modular product platforms", ASME press, New York, 1999.

[Dur11] D'Urso, P. I.; Lanzino, G.; Cloft, H.; Kallmes, D.: "Flow diversion for intracranial aneurysms: a review", in: Stroke, Vol. 42, No. 8, 2011, S. 2363-2368

[Gas05] Gasparotti, R.; Liserre, R.: "Intracranial aneurysms", in: European radiology, Vol. 15, No. 3, 2005, S. 441-447. doi: 10.1007/s00330-004-2614-8.

[Hac10] Hacke, W.: Neurologie, Springer Berlin Heidelberg, Berlin, Heidelberg, 2010.

[Hac20] Hackl, J.; Krause, D.; Otto, K. et al.: „Impact of Modularity Decisions on a Firm's Economic Objectives". Journal of Mechanical Design, Vol. 142/041403-1, 2020

[Han18] Hanna, M.; Schwede, L-N.; Krause, D. (2018): Model-Based Consistency for Design for Variety and Modularization. DSM 2018, Trieste.

[Har06] Harlou, U.: "Developing product families based on architectures. Contribution to a theory of product families", Technical University of Denmark, Lyngby, 2006.

[Hö106] Hölttä-Otto, K.; Otto, K.: "Platform Concept Evaluation": "Product Platform and Product Family Design. Methods and Applications", Springer, New York, 2006.

[Kra18] Krause, D., Gebhardt, N.: Methodische Entwicklung modularer Produktfamilien: Hohe Produktvielfalt beherrschbar entwickeln. Springer, Hamburg.

[Kuh20] Kuhl, J.; Ngo, N.; Buhk, J.-H.; Ding, A.; Braschkat, A.; Fiehler, J.; Krause, D.: Qualification of Additively Manufactured Blood Vessel Models for the Evaluation of Braided Stent Designs, in: Industrializing Additive Manufacturing - AMPA2020. 2020.

[Kvi10] Kvist, M.: "Product Family Assessment", Ph.D. thesis, Department of Management Engineering, Technical University of Denmark, Lyngby, 2010.

[Rip19] Ripperda, S.: "Methodische Unterstützung zur kostenbasierten Auswahl modularer Produktstrukturen", Produktentwicklung \& Konstruktionstechnik, Vol.15,Springer, 2019.

[Sa107] Salvador, F.: "Towards a Product System Modularity Construct: Literature Review and Reconceptualization", IEEE Transactions on Engineering Management, Vol. 54 (2), 2007.

[Sch20] Schwede, L. N.; Greve, E.; Krause, D.: "Validation Concept For The Investigation Of Effects Of Modular Product Families", Proceedings of DESIGN 2020, Dubrovnik, 2020.

[Sei19] Seiler, F; Schwede, L.-N.; Krause, D.: MBSE-basierte Produktkonfiguratoren zur Analyse der Modularisierung. EEE Conference, Vol. 2, Dresden. 2019

[Sei20] Seiler, F.; Krause, D.: „A Multi-Dimensional Configuration Algorithm For Modular Product Architectures, Proceedings of DESIGN 2020, Dubrovnik, 2020.

[Sim12] Simpson, T.W.; Bobuk, A.; Slingerland, L.A.; Brennan, S.; Logan, D.; Reichard, K.: "From user requirements to commonality specifications: an integrated approach to product family design", Research in Engineering Design, Vol. 23, 2012, pp. 141-153.

[War13] Wartzack, S.: "Auswahl und Bewertungsmethoden", in: Pahl, G.; Beitz, W.; Feldhusen, J.; Grote, K.-H. (Ed.): "Pahl/Beitz Konstruktionslehre: Methoden und Anwendung erfolgreicher Produktentwicklung", Springer, Berlin, 2013, pp. 380-409.

[Win19] Windheim, M.: Cooperative Decision-Making in Modular Product Family Design. Ph.D. thesis, Produktentwicklung \& Konstruktionstechnik, Vol. 17, Springer, 2019

[Yu07] Yu, T. L., Yassine, A. A., \& Goldberg, D. E. (2007). An information theoretic method for developing modular architectures. Research in Engineering Design, 18(2), 91-109.

Contact: F. M. Seiler, Hamburg University of Technology, Institute for Product Development and Mechanical Engineering Design, Denickestr. 17, 21073 Hamburg, Germany, florian.seiler@tuhh.de 